\title{
Editorial
}

\section{Dengue Outbreak in 2019}

\author{
Quazi Tarikul Islam ${ }^{1}$
}

\begin{abstract}
(c) (i) (ㅇ) C. $\mathrm{BY}$ NC SA

DOI: https://doi.org/I0.3329/jom.v2lil.44092

Copyright: (C) 2020 Islam QT. This is an open access article published under the Creative Commons Attribution-NonCommercial-NoDerivatives 4.0 International License, which permits use, distribution and reproduction in any medium, provided the original work is properly cited, is not changed in any way and it is not used for commercial purposes.
\end{abstract}

Received: 02 November, 2019;

Accepted: 18 November, 2019

Dengue is the most rapidly spreading mosquito-borne viral disease in the world. In the last 50 years, incidence has increased 30-fold with increasing geographic expansion to new countries and in the present decade, from urban to rural settings. An estimated 50 million dengue infections occur annually and approximately 2.5 billion people live in dengue endemic countries. ${ }^{1}$

Annually, 390 million people become infected with one of four serologically distinct serotypes of dengue virus (DENV1 to -4$)$, through the bites of infected females of Aedes aegypti ${ }^{2}$ This neglected tropical disease (NTD) including its severe form, dengue haemorrhagic fever have already predicted a dramatic increase of dengue in the following 30 years. ${ }^{3}$ Previous studies indicate that dengue may have seasonal and inter-annual patterns of occurrence.

Dengue transmission dynamics can be highly heterogeneous due to the complex interactions among virus serotypes, vector, and host. Some studies have associated these periodic outbreaks with global inter-annual climatic variations such as El Niño Southern Oscillation (ENSO). ${ }^{4}$ With the climate change there is established correlation of spread of Dengue is now a fact. In the tropical countries specially in South Asian region increase of 2 degree temperature there is increase incidence of Dengue. The duration of developmental time of adult mosquito is 30 days in 15 degree Celsius shorten to 8 days in temperature of 30 degree. Two decades before it was a disease affecting mostly children but presently Dengue is the disease affecting mostly the adults specially in Bangladesh, Indonesia, Singapore and Thailand.

The epidemiology of DF/ DHF is complex and remain poorly understood. It involve host, viral and vector status which is

1. Professor of Medicine, Popular Medical College and Editor in Chief, National Guideline for Clinical Management of Dengue Syndrome 2018. Email: prof.tarik@gmail.com. further influenced by demographic, economic, behavioral and varied societal factors. Dengue is one of the NTDs of major public health importance in Bangladesh, showing an increment in incidence, magnitude and frequency of outbreaks during the year 2019. From urban (Dhaka city) it had been spread to rural Bangladesh rather whole country. More than one hundred thousand cases have been recorded by the government though case fatality rate is less than 0.2 percent. $^{5}$

Largest dengue outbreak of the decade with high fatality may be due to reemergence of DEN-3 serotype in Dhaka, Bangladesh, necessitating immediate public health attention had been anticipated by IEDCR in November 2018. ${ }^{6}$ It happened so as largest outbreak of Dengue fever in the history of Bangladesh.

The case presentations were completely different, unusual, and unnatural than any year before. Majority cases present atypically. Myalgia, febrile rash, typical break bone pain were not the predominant features. Rather diarrhea, abdominal pain, ascites, pleural effusion and pneumonia were much more common presentations in 2019 outbreak. Co infections were a new and life threatening problem in 2019 outbreak. Typhoid fever, Pneumonia and infective colitis were predominant. In Dengue fever splenomegaly was a new experience this year. Physicians should search for Enteric Fever or haemophagocytic lymphohistiocytosis in this situation. Quiet good number of NS1 negative cases diagnosed as Dengue fever which is much higher than normal acceptable range. Many cases presented with ascites and pleural effusion before the appearance of critical phase. These unusual presentations makes diagnosis and management difficult.

There is significant variance in clinical manifestations and severity of dengue infection in different age groups. DF has 
been known in Asia for more than a century, and its severity is largely age-dependent. This disease is mild in children and more severe in adults. Infants and children with DF have symptoms ranging from an undifferentiated fever to a mild febrile illness, sometimes associated with a rash. Older children and adults frequently suffer a more severe form with high fever, pain in various parts of the body, and a maculopapular rash. However, the infection is only rarely fatal with co-morbidities that are more likely to be present in adults than in children such as peptic ulcers and preexisting liver disease, CKD can aggravate the disease severity. Thus, proper management of dengue patients must consider the different age-specific clinical manifestations. ${ }^{7}$ Dengue patients with severe organ involvement such as liver, kidneys, brain, or heart have been increasingly reported. These organopathies may be associated with co-infections, co-morbidities, or complications of prolonged shock. Organopathies can modify clinical presentations of dengue disease and result in missed or delayed diagnosis. ${ }^{8}$

The factors that may have contributed to rapid changing epidemiology of DF/ DHF in South East Asia region are the challenges that need to be addressed in designing operational research and implementation strategies. Operational research is needed to answer research questions on how the efficacy, cost effectiveness, sustainability and scaling up of existing and promising new control methods can be enhanced. The major factors influencing changes in dengue Epidemiology and clinical presentations include: i) viral genotypes/subtypes with increased virulence; ii) lack of information on human population genetics and its relation with viral genome; iii) lack of information on vector ecology in micro climatic conditions; iv) viral load injected by infected mosquito; v) post infection natural immunity of the host and vi) time interval in sequential infection. ${ }^{2}$
We should prepare all sectors to combat the expected outbreak of 2020. Dengue is no more a disease of monsoon and urban. The whole country will face the problem of the disease throughout the year.

\section{References:}

1. World Health Organization. Global strategy for dengue prevention and control 2012-2020. Geneva: WHO, 2012. Available from: http://apps.who.int/iris/bitstream/10665/ 75303/1/9789241504034_eng.pdf [Last accessed on 15 Oct 2018].

2. Bhatt $\mathrm{S}$, et al. The global distribution and burden of dengue. Nature. 2013;496:504-507. doi: 10.1038/nature12060.

3. Messina $J$, et al. The many projected futures of dengue. Nature Reviews Microbiology. 2015;13:230-239. doi: $10.1038 /$ nrmicro3430.

4. Van Panhuis $\mathrm{W}$, et al. Region-wide synchrony and traveling waves of dengue across eight countries in Southeast Asia. Proceedings of the National Academy of Sciences. 2015;112:13069-13074. doi:10.1073/pnas. 1501375112.

5. Emergency diseases control center, DGHS, Government of People's Republic of Bangladesh, November 2019

6. Shirin T, Muraduzzaman AKM, Alam AN, Sultana S, Siddiqua M, Khan MH et al. Largest dengue outbreak of the decade with high fatality may be due to reemergence of DEN3 serotype in Dhaka, Bangladesh, necessitating immediate public health attention. New Microbes New Infect. 2019 Feb 16;29:100511. doi: 10.1016/j.nmni.2019.01.007.

7. Southeast Asian J Trop Med Public Health Vol. 48 (Supplement 1) 2017 AW SAJ 2017.indd 112 8/25/2560 BE 12:41 PM severity of dengue disease (Panpitpat et al, 2007; Tantawichien, 2015)

8. Islam, QT. (2019). Changing Epidemiological and Clinical pattern of Dengue in Bangladesh 2018. Journal of Medicine 20(1):1-3. 